\title{
Soluble Receptor Tyrosine-Protein Kinase erbB-2
}

National Cancer Institute

\section{Source}

National Cancer Institute. Soluble Receptor Tyrosine-Protein Kinase erbB-2. NCI

Thesaurus. Code C18435.

Soluble receptor tyrosine-protein kinase erbB-2 is encoded by the human ERBB2 gene.

This protein may be involved in binding to receptor tyrosine-protein kinase erbB-2, which leads to disruption of dimer formation, reduced receptor phosphorylation, and inhibition of cell growth. 